\title{
Morphological Factor Estimation via High-Dimensional Reduction: Prediction of MCI Conversion to Probable AD
}

\author{
Simon Duchesne ${ }^{1,2}$ and Abderazzak Mouiha ${ }^{2}$ \\ ${ }^{1}$ Départment de Radiologie, Faculté de Mdecine, Université Laval, Québec, Canada G1K 7P4 \\ ${ }^{2}$ Centre de Recherche Université Laval Robert-Giffard, Québec, Canada G1J 2 G3 \\ Correspondence should be addressed to Simon Duchesne, simon.duchesne@crulrg.ulaval.ca
}

Received 24 December 2010; Accepted 27 April 2011

Academic Editor: Katsuya Urakami

Copyright (C) 2011 S. Duchesne and A. Mouiha. This is an open access article distributed under the Creative Commons Attribution License, which permits unrestricted use, distribution, and reproduction in any medium, provided the original work is properly cited.

We propose a novel morphological factor estimate from structural MRI for disease state evaluation. We tested this methodology in the context of Alzheimer's disease (AD) with 349 subjects. The method consisted in (a) creating a reference MRI feature eigenspace using intensity and local volume change data from 149 healthy, young subjects; (b) projecting MRI data from 75 probable AD, 76 controls (CTRL), and 49 Mild Cognitive Impairment (MCI) in that space; (c) extracting high-dimensional discriminant functions; (d) calculating a single morphological factor based on various models. We used this methodology in leave-one-out experiments to (1) confirm the superiority of an inverse-squared model over other approaches; (2) obtain accuracy estimates for the discrimination of probable AD from CTRL (90\%) and the prediction of conversion of MCI subjects to probable AD (79.4\%).

\section{Introduction}

A growing body of literature relates the use of machine learning methods to build classification functions from features of interest extracted from medical imaging data (e.g., magnetic resonance images (MRI), positron emission tomography). We focus specifically on applications within the context of aid to clinical diagnostic in Alzheimer's disease $(\mathrm{AD})$ and/or the prediction of future clinical status for individuals with Mild Cognitive Impairment (MCI), a putative precursor to $\mathrm{AD}[1-6]$. These techniques have in common the reduction of large, high-dimensional image vectors into smaller features spaces and the identification of a low-dimensional discriminating function. Authors have reported attempts to further simplify the discriminating function by calculating a single, quantitative scalar measure, for example, the structural abnormality index score [7], the structural-functional biomarker score [8], and the disease evaluation factor [9]. While the first two rely on support vector machine analysis of a feature space composed of grey matter concentration patterns, the latter relies on linear kernel approaches to data reduction and classification of MRI appearance, defined as the combination of T1-weighted intensity and local volume shape characteristics for all voxels within a volume of interest. Using the same features of interest as described in [9], we propose a different morphological factor formulation, extensible to other modalities and to other sources of data. We derive the formulation and estimate its efficiency within the context of aid to diagnostic in probable $\mathrm{AD}$ by verifying the hypothesis that it accurately describes current and future clinical status.

\section{Methods}

2.1. Subjects. A total of 349 subjects were included in this study, with ethics approval obtained from each institution represented.

The first cohort, or reference group, consisted in 149 young, neurologically healthy individuals obtained with permission from the ICBM database [10], whose scans were used to create a reference feature space of image data.

The second cohort, or $A D$ test group, consisted in 150 subjects: 75 patients with a diagnosis of probable $\mathrm{AD}(A D$ group) and 75 age-matched controls (CTRL group) without neurological or neuropsychological deficit. The probable $\mathrm{AD}$ subjects were individuals with mild to moderate probable 
$\mathrm{AD}$ [11] recruited among outpatients seen at the IRCCS Fatebenefratelli (Brescia, Italy) between November 2002 and January 2005. CTRL subjects were taken from an ongoing study of the structural features of normal aging at the same center [12]. All subjects were followed a minimum of 3 years after inclusion; this longitudinal clinical evaluation constitutes our reference diagnostic.

The third cohort, or MCI test group, consisted in 49 MCI subjects taken from a prospective project on the natural history of MCI, carried out at the IRCCS Fatebenefratelli. The project was aimed to study the natural history of nondemented persons with apparently primary cognitive deficits, that is, deficits not due to psychic (anxiety, depression, etc.) or physical (hypothyroidism, vit. B12 and folate deficiency, uncontrolled heart disease, uncontrolled diabetes, etc.) conditions. Patients were rated with a series of standardized diagnostic and severity instruments, including the Mini-Mental State Examination (MMSE; [13]). In addition, patients underwent diagnostic MRI and laboratory testing to rule out other causes of cognitive impairment. These inclusion and exclusion criteria for MCI were based on previous seminal studies [14-16]. Amnestic or nonamnestic, single or multiple domain MCIs were included in the study.

All MCI patients underwent a yearly follow-up visit, consisting of complete clinical and neuropsychological examination, from 1 to 4 years after enrolment. In those individuals that converted to dementia, status was ascertained according to clinical diagnostic criteria for AD [11], subcortical vascular dementia [17], dementia with Lewy bodies [18], and frontotemporal dementia [19]. Within the larger prospective cohort of $100 \mathrm{MCI}$ patients enrolled from April 2002 to December 2006, we have selected patients retrospectively for this study based on their (a) having been followed clinically a minimum of 48 months after their baseline MR scan; (b) having remained either stable (MCI-S group; $N=29$ ) or progressed to probable AD (MCI-P group; $N=20$; mean progression $1.5 \mathrm{yrs}$; SD $0.7 \mathrm{yrs}$ ). The 48 -month longitudinal clinical evaluation constitutes our reference diagnostic.

Data for the last subject (validation subject) was obtained with permission from the pilot, multicentric European ADNI project [20] (E-ADNI). It consisted in a healthy volunteer that acted as human quality control phantoms and that was scanned three times at IRCCS Fatebenefratelli (scan; repeat scan, same session; rescan) on the same day.

Ethics Committees approved the study, and informed consent was obtained from all participants.

2.2. Data. The ICBM subjects from the reference group were scanned in Montreal, Canada on a Philips Gyroscan 1.5T scanner (Best, Netherlands) using a T1-weighted fast gradient echo sequence (sagittal acquisition, $\mathrm{TR}=18 \mathrm{~ms}$, $\mathrm{TE}=10 \mathrm{~ms}, 1 \mathrm{~mm} \times 1 \mathrm{~mm} \times 1 \mathrm{~mm}$ voxels, flip angle $\left.30^{\circ}\right)$. MRI data for probable AD, CTRL, MCI, and E-ADNI subjects were acquired at the IRCCS Fatebenefratelli on a single Philips Gyroscan 1.0T scanner (Best, Netherlands) using a T1-weighted fast field echo sequence (sagittal acquisition, $\mathrm{TR}=25 \mathrm{~ms}, \mathrm{TE}=6.9 \mathrm{~ms}, 1 \mathrm{~mm} \times 1 \mathrm{~mm} \times 1,3 \mathrm{~mm}$ voxels $).$
2.3. Data Processing. We provide an overview of the automated image processing methodology, which follows essentially the steps outlined in Duchesne et al. with some modifications [3]. Images from all subjects were processed in an identical fashion using a publicly available toolkit (MINC: http://www.bic.mni.mcgill.ca/ServicesSoftware/HomePage). Processing included intensity inhomogeneity correction [21], nonlocal means denoising [22], intensity scaling, global and linear registration [23], extraction of a predetermined volume of interest centered on the medial temporal lobes, nonlinear registration within the volume of interest towards a common reference target [24], and computation of logdeterminants of the Jacobian of the deformation field [25].

2.4. Data Reduction and Feature Selection. The first data reduction step was to construct a feature space based on the $N=149$ subjects from the ICBM reference group. To this end, we used Principal Components Analysis (PCA) of two high-dimensional image vectors within a volume of interest centered on the medial temporal lobe to generate a low-dimensional feature space for classification: (1) the T1-weighted MRI intensity within the volume of interest, transformed into $z$-score; and (2) log-determinants within the volume of interest. With PCA we moved from a massive amount of data $\left(2 \times 149 \times 4 E 10^{5}\right.$ voxels $)$ to a lower subspace model of maximum $N-1$ dimensionality, further restricted by using only the first $k$ eigenvectors $\lambda$ that contribute up to a given threshold $r$ in the description of the total variance of the system:

$$
r_{k}=\frac{\lambda_{k}}{\sum_{j=1}^{p} \lambda_{j}} .
$$

Once the reference eigenspace was formed, the reference group data was no longer used.

We then proceeded by projecting rasterized vectors of intensity and local volume changes for subjects in the $A D$ and $M C I$ test groups into the reference space. The distribution of eigencoordinates along any principal component for a given population was assessed via quantile plots and Shapiro-Wilke statistics for normal distribution. Following the projection, we used a system of supervised linear classifiers to identify the hyperplane that best separated the groups under study (e.g., CTRL versus probable AD; MCI-S versus MCI-P). To this end, the data was first normalized to guard against variables with larger variance that might otherwise dominate the classification. We employed forward stepwise regression analysis via Wilk's $\lambda$ method to select the set of discriminating variables $\left\{\lambda_{F}\right\}$, with $F \ll N-1$, forming the discriminating hyperplane.

2.5. Comparative Morphological Factor Construction. The morphological factor is based on the concept of distance along the restricted set of eigenvectors $\left\{\lambda_{F}\right\}$. In the imagebased feature space, this distance $d$ can be calculated in a number of different fashions.

2.5.1. Manhattan Distance. We propose initially the signed difference between subject eigencoordinates along the eigenvector $\lambda_{F}$ and the mean of the CTRL distribution for that 
eigenvector, denoted $\bar{m}_{\mathrm{CTRL}}$; as this distance increases the likelihood of belonging to the CTRL group decreases:

$$
d_{i}^{\lambda_{F}}=x_{i}^{\lambda_{F}}-\bar{m}_{\mathrm{CTRL}}^{\lambda_{F}} .
$$

2.5.2. Euclidean Distance. We propose the Euclidean distance between position $p_{i}$ of each subject $s_{i}$ and both CTRL and probable $\mathrm{AD}$ means along the restricted set of eigenvectors $\left\{\lambda_{F}\right\}$ in all $\mathrm{F}$ directions, with $F \ll N-1$. As the distance to one center decreases, the distance to the second should increase. In (3) we demonstrate the distance to the mean of the probable $\mathrm{AD}$ group:

$$
d_{s_{i} \rightarrow \mathrm{CM}_{\mathrm{AD}}}=\sqrt{\sum_{F}\left(p_{i}^{f}-\bar{m}_{\mathrm{AD}}^{f}\right)^{2}} .
$$

2.5.3. Weighted Distance. It is possible to weigh each eigenvector by an associated measure of significance, for example, Wilk's $\lambda$ from the stepwise regression analysis [9] or a factor derived from univariate $t$-tests. While the Wilk's $\lambda$ is trivially obtained from the regression analysis, an univariate weight such as the Koikkalainen factor formulation [26] entails performing a $t$-test comparing the group eigencoordinate distributions (e.g., CTRL versus probable AD; MCI-S versus MCI-P) for each eigenvector of the restricted set, resulting in the $P$-value $p\left(\lambda_{F}\right)$ for that distribution; from these $P$-values the significance weight $S_{F}$ is calculated,

$$
S_{F}=\frac{\ln \min \left[p\left(\lambda_{F}\right), 0.05\right]-\ln 0.05}{\ln 0.000001-\ln 0.05} .
$$

The significance increases as the differences between the CTRL and AD groups grow and reaches zero when there is no statistically significant difference (at the $P=.05$ level) between both distributions.

The resulting weighted distance $D_{i}$ combines the aforementioned distances (Manhattan, Euclidean) with a weight $S_{F}$ (either Wilk's $\lambda$ or Koikkalainen factor) over all eigenvectors $F$ from the restricted set $\left\{\lambda_{F}\right\}$ as follows:

$$
D_{i}=\frac{\sum_{i}^{\lambda_{F}} S_{F} d_{i}^{\lambda_{F}}}{\sum_{\lambda_{F}} S_{F}}
$$

2.5.4. Gravitational Model. As the final formulation, we extend the principle of image-based distance to the context of an attraction field that follows Netwon's Law of Universal Gravitation, whereby any two elements of mass $m$ within the feature space will exert upon one another an attractive force that will vary proportionally to the inverse of the square of the distance between them. In our context the force exerted by one group (e.g., CTRL) decreases as the distance between a subject and the center of mass of the CTRL group grows, while the force exerted by the second group (e.g., probable $\mathrm{AD}$ ) increases as distance decreases between the same subject and the second group's center of mass. In a multiple group scenario, the calculated combined force serves as a quantitative measure of the likelihood of belonging to one of the groups.
In such a classical formulation the force between any subject $s_{i}$ with mass $m_{i}$, to the centers of mass of, for example, the CTRL group $\left(\mathrm{CM}_{\mathrm{CTRL}}\right)$ and the $\mathrm{AD}$ group $\left(\mathrm{CM}_{\mathrm{AD}}\right)$, is expressed as:

$$
F_{s_{i} \rightarrow \mathrm{CTRL}, \mathrm{AD}}=G m_{i}\left(\frac{\mathrm{CM}_{\mathrm{CTRL}}}{d_{s_{i} \rightarrow \mathrm{CM}_{\mathrm{CTRL}}}^{2}}-\frac{\mathrm{CM}_{\mathrm{AD}}}{d_{s_{i}}^{2} \rightarrow \mathrm{CM}_{\mathrm{AD}}}\right)
$$

with

$$
\mathrm{CM}=\frac{1}{M} \sum_{i} m_{i} p_{i},
$$

being the formulation for the centers of mass calculations, where $M$ is the total mass for all subjects in the group, $m_{i}$ their individual masses, and $p_{i}$ their individual positions in feature space as derived in the previous section. The distance metric that can be used can be anyone of the aforementioned distances; for the purposes of the current study, the Euclidean distance as formulated in (3) was employed.

We chose to retain the concept of "mass" even though it has no real bearing within the present context of an imagebased feature space. It could be replaced with different information regarding individuals in the groups, for example, Braak histopathological staging [27]. Alternatively, one can vary the specificity and sensitivity of the attraction field by increasing the "mass" of subjects in one of the groups (e.g., CTRL or probable AD). For these purposes however we set the mass of each subject to unity, and, further, for equal considerations of simplicity, we set the gravitational constant $G$ also to unity. As is, the result is an inverse-squared law relationship.

Statistics and measurements were computed using the MATLAB Statistics Toolbox (The MathWorks, Natick, MA).

2.6. Experiments. Once the reference space was created, all of the experiments that we conducted were performed in a leave-one-out fashion whereby one subject from the study groups was temporarily removed, allowing for an independent estimate of the low-dimensional discriminant function and the calculation of the eigendistribution means and centers of mass. Only then was the left-out subject entered in the system and its morphological factor computed. The final results consist in the comparison of the independently acquired morphological factors for each subject.

We ran three distinct experiments: (a) determination of the relative accuracies of each distance formulation (Manhattan, Euclidean, Weighted Distance (Wilk's $\lambda$ ), Weighted Distance (Koikkalainen), Gravitational model) for the discrimination of CTRL versus probable AD; (b) determination of the accuracy of the best distance formulation for the discrimination of MCI-S versus MCI-P; (c) determination of the resolution of the best distance formulation based on the CTRL versus probable $\mathrm{AD}$ discriminant function using the E-ADNI scan-rescan dataset.

\section{Results}

3.1. Demographics. There were no statistically significant differences for age between the 75 probable AD and $75 \mathrm{NC}$ 
TABle 1: Demographic information.

\begin{tabular}{lcccc}
\hline & CTRL & AD & MCI-S & MCI-P \\
\hline$N$ & 75 & 75 & 29 & 20 \\
Age (mean, SD) & $73.3(4.6)$ & $73.3(8.4)$ & $63.5(14.2)$ & $74.2(6.3)$ \\
Sex & $23 \mathrm{M} ; 52 \mathrm{~F}$ & $15 \mathrm{M} ; 60 \mathrm{~F}$ & $9 \mathrm{M} ; 20 \mathrm{~F}$ & $10 \mathrm{M} ; 10 \mathrm{~F}$ \\
Baseline MMSE (mean, SD) & & & $27.7(1.5)$ & $26.4(1.6)$ \\
\hline
\end{tabular}

TABLE 2: Model results.

\begin{tabular}{lccccc}
\hline & Gravity & \multicolumn{2}{c}{ Weighted distance } & Euclidean & Manhattan \\
& & Koikkalainen & Wilk's $\lambda$ & 0.85 & 0.73 \\
\hline Accuracy & 0.90 & 0.86 & 0.78 \\
\hline
\end{tabular}

TABLE 3: Morphological factor results.

\begin{tabular}{lcccc}
\hline & CTRL & AD & MCI-S & MCI-P \\
\hline$N$ & 75 & 75 & 29 & 20 \\
Mean & 0.61 & -0.01 & 0.45 & 0.24 \\
Std dev & 0.32 & 0.23 & 0.26 & 0.27 \\
Std Err mean & 0.04 & 0.03 & 0.05 & 0.06 \\
Upper 95\% mean & 0.68 & 0.04 & 0.55 & 0.37 \\
Lower 95\% mean & 0.53 & -0.06 & 0.35 & 0.12 \\
\hline
\end{tabular}

individuals $(P>.05)$ in the $\mathrm{AD}$ test group. There was a statistical difference for age between the MCI-S and MCI$\mathrm{P}$ groups $(P=.001)$ and for baseline $\operatorname{MMSE}(P=.01)$ (see Table 1).

3.2. Data Processing and Feature Selection. We set the variance ratio $r$ (see Equation (1)) to 0.997, resulting in a reference PCA model composed of 256 intensity and local volume change eigenvectors. We proceeded with forward stepwise regression analysis using Wilk's $\lambda$ method ( $P$-toenter $=.005)$ to select the discriminating variables forming the hyperplane separating each group (e.g., CTRL versus probable AD; MCI-S versus MCI-P). This was performed in a leave-one-out fashion to eliminate overlearning of the dataset. The median number of eigenvectors $\lambda_{F}$ retained in the discriminating function for either CTRL versus probable $\mathrm{AD}$ or MCI-S versus MCI-P was four.

3.3. Morphological Factor Calculation. Table 2 displays the different accuracies obtained for the five different formulations for the morphological factor at the task of discriminating CTRL versus probable AD (leave-one-out). The Gravitational model's accuracy was $90 \%$, superior to the Weighted Distance models.

Using the Gravitational model, we report the results for the morphological factor for the CTRL versus probable AD experiment and the MCI-S versus MCI-P experiment in Table 3. The distributions of morphological factors for all groups, alongside quantile plots to assess normality (CTRL and probable AD groups) are shown in Figures 1 and 2.

The receiver operating characteristic (ROC) curve for the task of discriminating CTRL from probable AD shows the trade-offs possible in sensitivity and specificity (Figure 1(c)). The area under the ROC curve was 0.9444 . At the $90 \%$ accuracy point (135/150), specificity was $87.5 \%$ and sensitivity $92.9 \%$.

With the Gravitational model we computed the ROC curve for the discrimination of MCI-S from MCI-P (Figure 2(c)). The Area under the ROC curve was 0.7940. At $72.3 \%$ accuracy, specificity was $62 \%$ and sensitivity $75 \%$.

Finally, we computed the morphological factor for the EADNI human phantom volunteer, using the CTRL and probable $\mathrm{AD}$ cohorts as a training group for the determination of the discriminating function. Using the Gravitational model, the average factor value was -0.4 or 4 standard deviations away from the mean of the CTRL distribution, with an average difference in scan-rescan factor of $4 \%$. Notably, the morphological index obtained via a weighted distance method (Koikkalainen factor) had an average difference in scan-rescan factor of less than $1 \%$.

\section{Discussion}

The gravitational or inverse-squared law model constitutes a novel development in the strategies towards obtaining a single quantitative factor from data reduction and machine learning of very high-dimensional MRI input data towards 


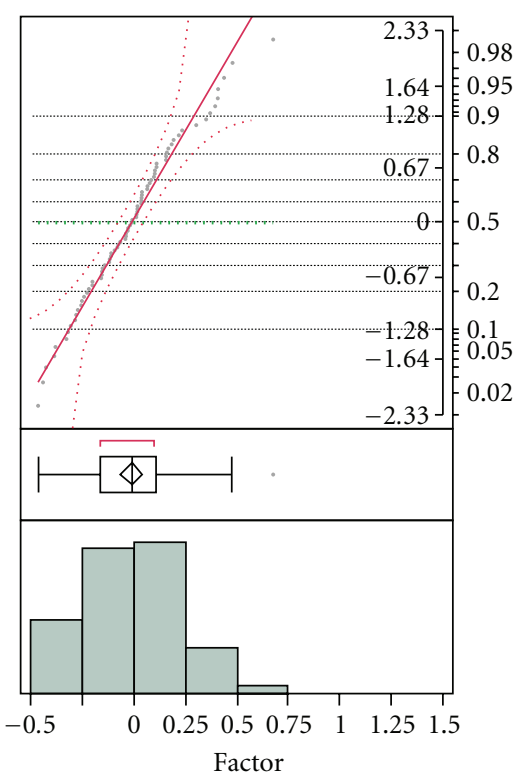

(a)

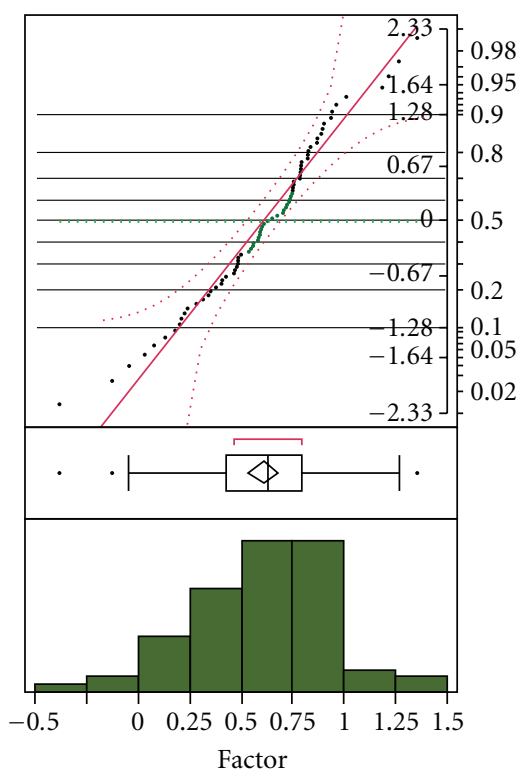

(b)

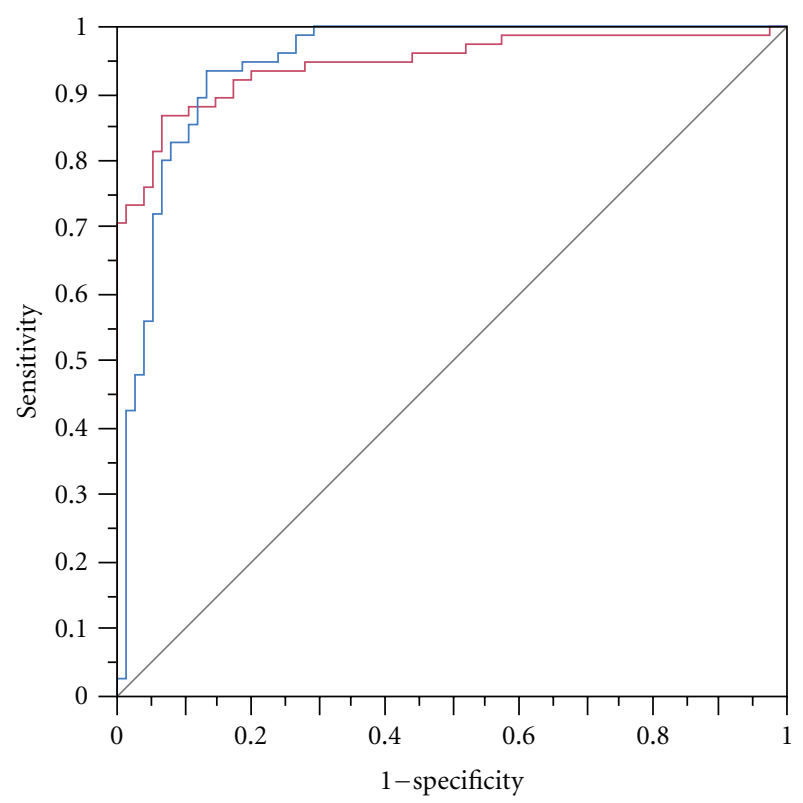

(c)

FIgURE 1: (a, b) Distributions of morphological factors for the CTRL (a) and probable AD groups (b) alongside quantile plots based on the Gravitational model (see Section 2.5.4). (c) Receiver operating characteristic curve (ROC) for the morphological factor displaying the trade-offs between sensitivity and specificity at the task of discriminating CTRL versus probable AD. The area under the ROC curve was 0.9444 . At the $90 \%$ accuracy point (135/150), specificity was $87.5 \%$ and sensitivity $92.9 \%$.

discrimination of individual subjects. Its inherent flexibility makes multigroup comparisons trivial, alongside the introduction of other sources of data. Its performance compares favorably to other results in the MRI literature within the context of discriminating CTRL versus probable AD [2]. As a single dimensional scalar, the morphological factor metric achieves strong accuracy (90\%), especially when compared to other multidimensional discrimination functions (e.g., $92 \%$ as reported in [3]). It has also a strong result when put within the clinical context of discriminating CTRL versus probable $\mathrm{AD}$, where inclusion evaluations are reportedly $78 \%$ accurate (albeit against final histopathological diagnostic). While lower, accuracy figures for the prediction of progression to probable $\mathrm{AD}$ in the $\mathrm{MCI}$ cohort (on average, 1.5 years before clinical diagnostic) are also strong and compare favorably to published results on MRI data $[4,6]$. A study comparing these approaches (e.g., within a monocentric setting, such as the Open Access Series of 


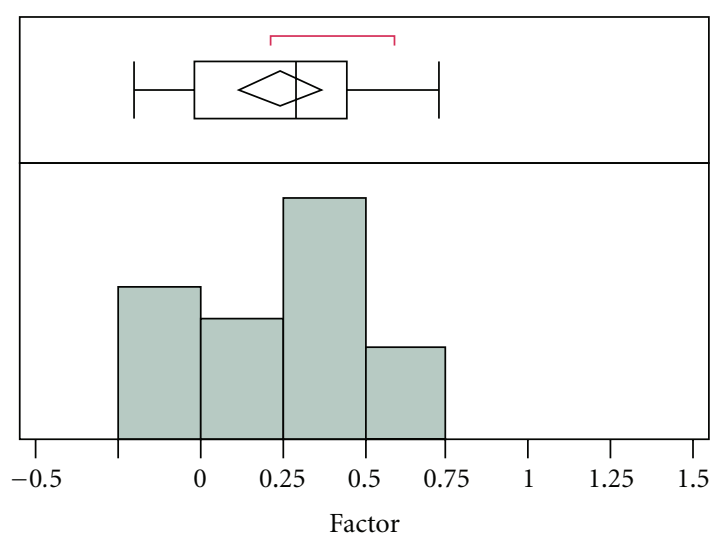

(a)

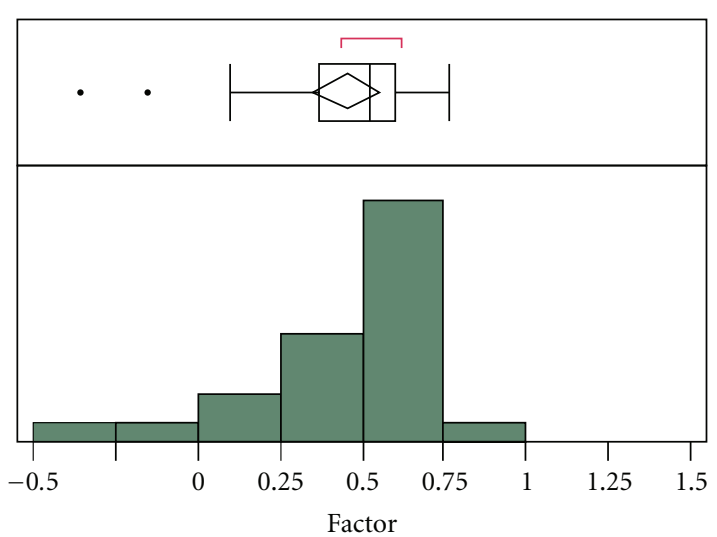

(b)

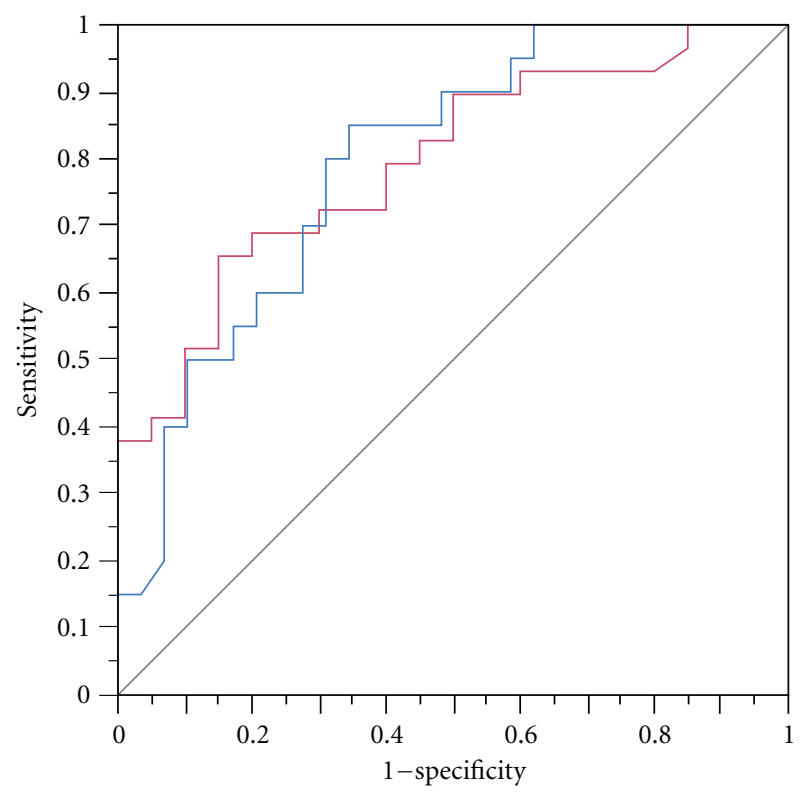

(c)

FIgure 2: (a, b) Similar distributions of morphological factors for the MCI-S (a) and MCI-P groups (b). (c) ROC for the discrimination of MCI-S and MCI-P. The area under the ROC curve was 0.7940 . At $72.3 \%$ accuracy, specificity was $62 \%$, and sensitivity $75 \%$.

Imaging Studies [28] or multicentric setting such as the Alzheimer's Disease Neuroimaging Initiative [29]) would be worthwhile.

The paper uses the leave-one-out approach to feature selection (stepwise regression analysis), which allows a correct generalization of the morphological factor as it is not tested on the same data.

Clinical interpretation of changes in image features associated with changes in the morphological factor should provide insight into the development of $\mathrm{AD}$ and would need to be compared to existing results from voxel-based morphometry studies, structural studies (e.g., hippocampal and entorhinal atrophy), and histopathological confirmation studies. Overall, we speculate that the specific patterns of intensity and local volume change differences result from different levels of advanced extracellular plaque formation, neurofibrillary tangles accumulation and other pathological processes between CTRL and probable $\mathrm{AD}$, and between stable and progressing MCI. With regards to the features employed in this method, the differences in local volume changes should mirror the changes noticed in other reports, such as visual assessment [30], while differences in grey level might reflect the intensity of neuronal loss induced by the neuropathological changes [31], which precede volume loss as visualized on MRI. Such an evaluation however is beyond the scope of this paper.

The difference in factor averages between probable $\mathrm{AD}$ and CTRL was $15 \%$. At this level, the minimum trial size required to detect this difference is 59 individuals for both samples $(\alpha=0.05 ; \beta=0.50)$ and reaches 75 individuals if we include scan-rescan variability.

4.1. Limitations. There are a number of limitations in this study. One pertains to the fact that the MRI images for the 
probable $\mathrm{AD}$ subjects were acquired at the time of diagnosis; therefore, some of the patients have had AD for a number of years. In turn, this implies that extensive neurodegeneration has taken place at this point and should artificially facilitate the discrimination with CTRL. However, the fact that the latter were age matched and the fact that the results in the MCI cohort remain significant alleviate part of this concern. It would be useful to assess if the morphological factor correlates with different indices of disease severity, cognitive deficits, or other biomarkers. Neuropathological confirmation is also required to replace the clinical evaluation as a gold standard. Finally, the patterns of abnormalities that can be found by the method are restricted to a space that is built from healthy, young controls. It is not the optimal space to describe normal aging and/or AD-related variability. However, it does tend to maximize the distance between both groups, as we noticed from building a few reference spaces in an $\mathrm{N}$-fold validation of the CTRL/probable AD groups that achieved lower accuracies.

We estimate that the proposed formulation of the morphological factor is relevant within the context of aid to diagnostic and prediction of future clinical status in probable $\mathrm{AD}$.

\section{Acknowledgments}

The authors thank the Laboratory for Epidemiology, Neuroimaging, and Telemedicine (LENITEM, IRCCS Fatebenefratelli, Brescia, Italy) (principal investigator: G. B. Frisoni) for access to their data. Their studies were supported by the Italian Ministry of Health, under Grant agreement "Archivio normativo italiano di morfometria cerebrale con risonanza magnetica (età 40+)," No. 00.343, and Grant agreement "Decadimento cognitivo lieve non dementigeno: stadio preclinico di malattia di Alzheimer e demenza vascolare. Caratterizzazione clinica, strumentale, genetica e neurobiologica e sviluppo di criteri diagnostici utilizzabili nella realtà nazionale," No. $533 \mathrm{~F} / \mathrm{B} / 1$. The authors thank the International Consortium for Brain Mapping and the pilot European ADNI study (PI: G. B. Frisoni) for their data. The authors' research was supported by funding from the Ministère du Développement Économique, de l'Innovation et de l'Exportation du Québec.

\section{References}

[1] Z. Lao, D. Shen, Z. Xue, B. Karacali, S. M. Resnick, and C. Davatzikos, "Morphological classification of brains via highdimensional shape transformations and machine learning methods," NeuroImage, vol. 21, no. 1, pp. 46-57, 2004.

[2] C. Davatzikos, Y. Fan, X. Wu, D. Shen, and S. M. Resnick, "Detection of prodromal Alzheimer's disease via pattern classification of magnetic resonance imaging," Neurobiology of Aging, vol. 29, no. 4, pp. 514-523, 2008.

[3] S. Duchesne, A. Caroli, C. Geroldi, C. Barillot, G. B. Frisoni, and D. L. Collins, "MRI-based automated computer classification of probable $\mathrm{AD}$ versus normal controls," IEEE Transactions on Medical Imaging, vol. 27, no. 4, Article ID 4479633, pp. 509-520, 2008.
[4] Y. Fan, N. Batmanghelich, C. M. Clark, and C. Davatzikos, "Spatial patterns of brain atrophy in MCI patients, identified via high-dimensional pattern classification, predict subsequent cognitive decline," NeuroImage, vol. 39, no. 4, pp. 1731-1743, 2008.

[5] S. Klöppel, C. M. Stonnington, C. Chu et al., "Automatic classi-fication of MR scans in Alzheimer's disease," Brain, vol. 131, no. 3, pp. 681-689, 2008.

[6] S. Duchesne, C. Bocti, K. De Sousa, G. B. Frisoni, H. Chertkow, and D. L. Collins, "Amnestic MCI future clinical status prediction using baseline MRI features," Neurobiology of Aging, vol. 31, no. 9, pp. 1606-1617, 2010.

[7] P. Vemuri, J. L. Gunter, M. L. Senjem et al., "Alzheimer's disease diagnosis in individual subjects using structural MR images: validation studies," NeuroImage, vol. 39, no. 3, pp. 1186-1197, 2008.

[8] Y. Fan, S. M. Resnick, X. Wu, and C. Davatzikos, "Structural and functional biomarkers of prodromal Alzheimer's disease: a high-dimensional pattern classification study," NeuroImage, vol. 41, no. 2, pp. 277-285, 2008.

[9] S. Duchesne, "Quantitative evaluation of Alzheimer's disease," in SPIE-Medical Imaging, SPIE Society, Orlando, Fla, USA, 2009.

[10] J. C. Mazziotta, A. W. Toga, A. Evans, P. Fox, and J. Lancaster, "A probabilistic atlas of the human brain: theory and rationale for its development : the International Consortium for Brain Mapping (ICBM)," NeuroImage, vol. 2, no. 2, pp. 89-101, 1995.

[11] G. McKhann, D. Drachman, and M. Folstein, "Clinical diagnosis of Alzheimer's disease: report of the NINCDS-ADRDA work group under the auspices of department of health and human services task force on Alzheimer's disease," Neurology, vol. 34, no. 7, pp. 939-944, 1984.

[12] S. Galluzzi, C. Testa, M. Boccardi et al., "The Italian brain normative archive of structural MR scans: norms for medial temporal atrophy and white matter lesions," Aging-Clinical and Experimental Research, vol. 21, no. 4-5, pp. 266-276, 2009.

[13] M. F. Folstein, S. E. Folstein, and P. R. McHugh, "“Mini mental state". A practical method for grading the cognitive state of patients for the clinician," Journal of Psychiatric Research, vol. 12, no. 3, pp. 189-198, 1975.

[14] R. C. Petersen, R. Doody, A. Kurz et al., "Current concepts in mild cognitive impairment," Archives of Neurology, vol. 58, no. 12, pp. 1985-1992, 2001.

[15] R. C. Petersen, "Mild cognitive impairment as a diagnostic entity," Journal of Internal Medicine, vol. 256, no. 3, pp. 183-194, 2004.

[16] B. Dubois, H. H. Feldman, C. Jacova et al., "Research criteria for the diagnosis of Alzheimer's disease: revising the NINCDS-ADRDA criteria," The Lancet Neurology, vol. 6, no. 8, pp. 734-746, 2007.

[17] T. Erkinjuntti, D. Inzitari, L. Pantoni et al., "Research criteria for subcortical vascular dementia in clinical trials," Journal of Neural Transmission, Supplement, no. 59, pp. 23-30, 2000.

[18] I. G. McKeith, C. G. Ballard, R. H. Perry et al., "Prospective validation of consensus criteria for the diagnosis of dementia with Lewy bodies," Neurology, vol. 54, no. 5, pp. 1050-1058, 2000.

[19] D. S. Knopman, B. F. Boeve, J. E. Parisi et al., "Antemortem diagnosis of frontotemporal lobar degeneration," Annals of Neurology, vol. 57, no. 4, pp. 480-488, 2005. 
[20] G. B. Frisoni, W. J. P. Henneman, M. W. Weiner et al., "The pilot European Alzheimer's disease neuroimaging initiative of the European Alzheimer's disease consortium," Alzheimer's and Dementia, vol. 4, no. 4, pp. 255-264, 2008.

[21] J. G. Sled, A. P. Zijdenbos, and A. C. Evans, "A nonparametric method for automatic correction of intensity nonuniformity in MRI data," IEEE Transactions on Medical Imaging, vol. 17, no. 1, pp. 87-97, 1998.

[22] P. Coupe, P. Yger, S. Prima, P. Hellier, C. Kervrann, and C. Barillot, "An optimized blockwise nonlocal means denoising filter for 3-D magnetic resonance images," IEEE Transactions on Medical Imaging, vol. 27, no. 4, Article ID 4359947, pp. 425-441, 2008.

[23] D. L. Collins, P. Neelin, T. M. Peters, and A. C. Evans, "Automatic 3D intersubject registration of $\mathrm{MR}$ volumetric data in standardized Talairach space," Journal of Computer Assisted Tomography, vol. 18, no. 2, pp. 192-205, 1994.

[24] D. L. Collins and A. C. Evans, "Animal: validation and application of nonlinear registration-based segmentation," International Journal of Pattern Recognition and Artificial Intelligence, vol. 11, no. 8, pp. 1271-1294, 1997.

[25] M. K. Chung, K. J. Worsley, T. Paus et al., "A unified statistical approach to deformation-based morphometry," NeuroImage, vol. 14, no. 3, pp. 595-606, 2001.

[26] J. Koikkalainen et al., "Estimation of disease state using statistical information from medical imaging data," in Medical Image Computing and Computer Assisted Intervention-From Statistical Atlases to Personnalized Models Workshop, MICCAI Society, Copenhagen, Denmark, 2006.

[27] H. Braak and E. Braak, "Neuropathological stageing of Alzhei-mer-related changes," Acta Neuropathologica, vol. 82, no. 4, pp. 239-259, 1991.

[28] D. S. Marcus, T. H. Wang, J. Parker, J. G. Csernansky, J. C. Morris, and R. L. Buckner, "Open access series of imaging studies (OASIS): cross-sectional MRI data in young, middle aged, nondemented, and demented older adults," Journal of Cognitive Neuroscience, vol. 19, no. 9, pp. 1498-1507, 2007.

[29] S. G. Mueller, M. W. Weiner, L. J. Thal et al., "Ways toward an early diagnosis in Alzheimer's disease: the Alzheimer's disease neuroimaging initiative (ADNI)," Alzheimer's and Dementia, vol. 1, no. 1, pp. 55-66, 2005.

[30] L. O. Wahlund, P. Julin, S. E. Johansson, and P. Scheltens, "Visual rating and volumetry of the medial temporal lobe on magnetic resonance imaging in dementia: a comparative study," Journal of Neurology Neurosurgery and Psychiatry, vol. 69, no. 5, pp. 630-635, 2000.

[31] L. O. Wahlund and K. Blennow, "Cerebrospinal fluid biomarkers for disease stage and intensity in cognitively impaired patients," Neuroscience Letters, vol. 339, no. 2, pp. 99-102, 2003. 


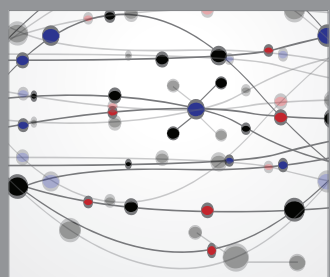

The Scientific World Journal
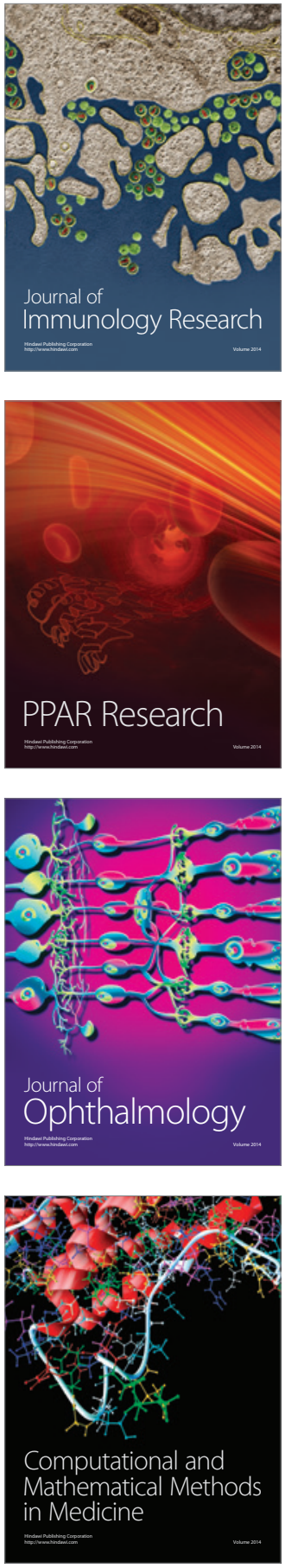

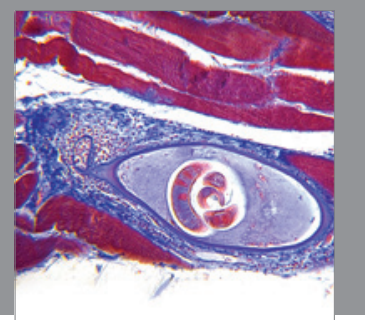

Gastroenterology

Research and Practice
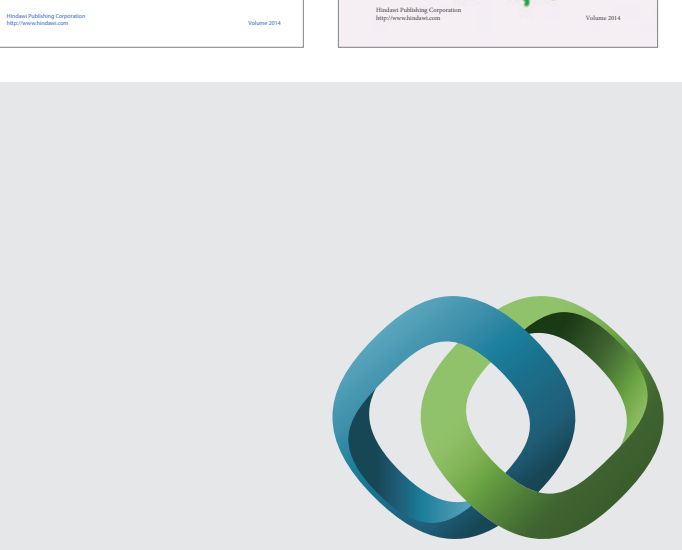

\section{Hindawi}

Submit your manuscripts at

http://www.hindawi.com
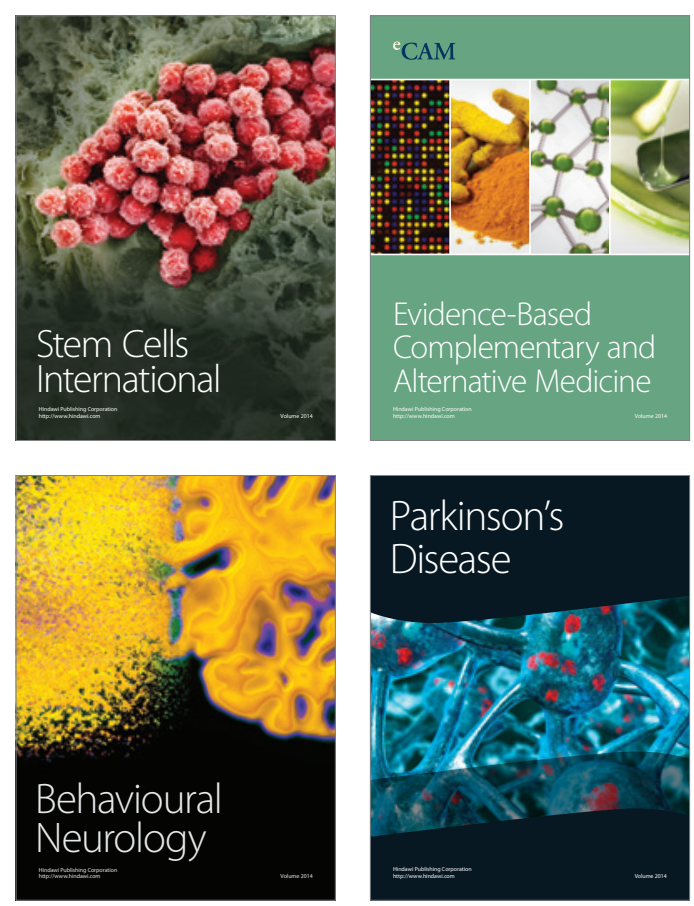

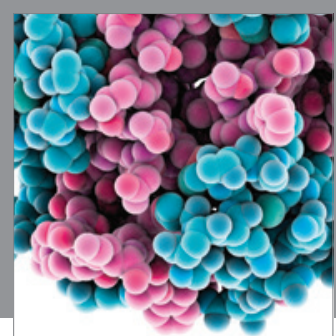

Journal of
Diabetes Research

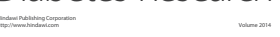

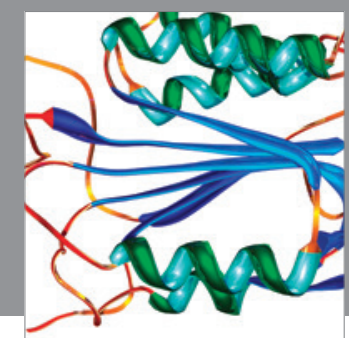

Disease Markers
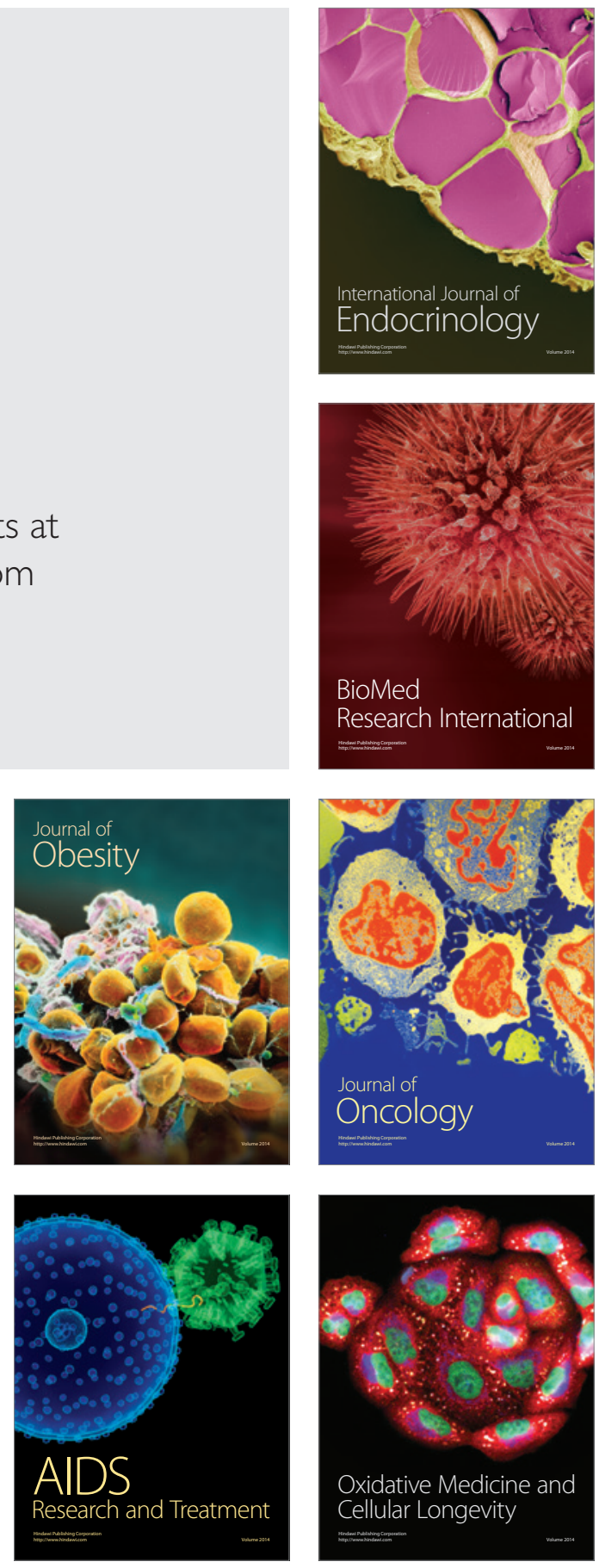